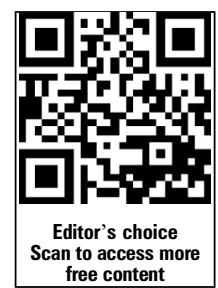

can to access m
free content

\title{
Self-guided internet-based and mobile-based stress management for employees: results of a randomised controlled trial
}

\author{
David Daniel Ebert, ${ }^{1,2}$ Elena Heber, ${ }^{2}$ Matthias Berking, ${ }^{1}$ Heleen Riper, ${ }^{2,3,4,5}$ \\ Pim Cuijpers, ${ }^{2,3,5}$ Burkhardt Funk, ${ }^{2}$ Dirk Lehr ${ }^{2}$
}

- Additional material is published online only. To view please visit the journal online (http://dx.doi.org/10.1136/ oemed-2015-103269).

1 Department of Clinical Psychology and Psychotherapy, Friedrich-Alexander-University Erlangen-Nuremberg, Erlangen, Bavaria, Germany

2Division of Online Health

Training, Innovation Incubator, Leuphana University, Lueneburg, Germany ${ }^{3}$ Department of Clinical Psychology, VU University, Amsterdam, The Netherlands ${ }^{4}$ Telepsychiatric Centre, University of Southern Denmark, Odense, Denmark Institute for Health and Care Research (EMGO), VU University Medical Centre, Amsterdam, The Netherlands

\section{Correspondence to} Dr David Daniel Ebert, Department of Clinical Psychology and Psychotherapy, Friedrich-Alexander University Erlangen-Nuremberg, Erlangen 90453, Germany;

david.ebert@fau.de

Received 20 August 2015 Revised 11 December 2015 Accepted 20 January 2016 Published Online First 16 February 2016

\section{ABSTRACT}

Objective This randomised controlled trial (RCT) aimed to evaluate the efficacy of a self-guided internet-based stress management intervention (iSMI) for employees compared to a 6-month wait-list control group (WLC) with full access for both groups to treatment as usual. Method A sample of 264 employees with elevated symptoms of perceived stress (Perceived Stress Scale, PSS-10 $\geq 22$ ) was randomly assigned to either the iSMI or to the WLC. The iSMI consisted of seven sessions and one booster session including problem-solving and emotion regulation techniques. Self-report data were assessed at baseline, at 7 weeks and at 6 months following randomisation. The primary outcome was perceived stress (PSS-10). The secondary outcomes included other relevant mental-related and work-related health outcomes. Data were analysed based on intention-to-treat principles.

Results The iSMI participants showed a significantly higher reduction in perceived stress from baseline to post-treatment at 7 weeks $(\mathrm{d}=0.96,95 \% \mathrm{Cl} 0.70$ to 1.21) and to the 6-month follow-up $(d=0.65,95 \% \mathrm{Cl}$ 0.40 to 0.89 ) compared to the WLC. Significant differences with small to moderate effect sizes were also found for depression, anxiety, emotional exhaustion, sleeping problems, worrying, mental health-related quality of life, psychological detachment, emotion regulation skills and presenteeism, in favour of the experimental group. At the 6 -month follow-up, all outcomes remained significantly better for the experimental group with the exception of work engagement, physical health-related quality of life and absenteeism, which were not found to significantly differ between the iSMI and WLC groups.

Conclusions The iSMI investigated in this study was found to be effective in reducing typical mental-related and work-related health symptoms of stressed employees. Internet-based self-guided interventions could be an acceptable, effective and potentially costeffective approach to reduce the negative consequences associated with work-related stress.

\section{BACKGROUND}

Work-related stress has become a major public health problem with serious consequences for the individual, companies and society. It is associated with increased risk for adverse health consequences, such as fatigue, sleeping problems, ${ }^{1}$ coronary disease $^{2}$ and mental health problems. ${ }^{3}$

\section{What this paper adds}

- Work-related stress is common but undertreated.

- Psychological interventions are effective, but evidence for long-term effectiveness is scarce.

- Internet-based self-help interventions are promising, but the few studies conducted to date on internet-based self-guided stress-management yield mixed results.

- The present study provides evidence for the (long-term) effectiveness of self-guided internet-based and mobile-supported stress management on a range of relevant mental health, work-related health and skill-related outcomes.

- Internet-based self-guided interventions could be an acceptable, effective and potentially cost-effective approach to reduce the negative consequences associated with work-related stress.

Moreover, stress is also associated with substantial economic costs due to absenteeism, staff turnover and reduced work performance. ${ }^{4}$

In the last decades, a number of interventions for occupational stress have been developed. The efficacy of these interventions has been demonstrated in a large number of randomised controlled trials (RCTs), ${ }^{5}{ }^{6}$ although evidence on the long-term effectiveness is scarce. ${ }^{6}$ In spite of the availability of evidence-based interventions, the majority of stressed employees remain untreated.

Using the internet to provide self-help interventions may aid in overcoming some of the limitations of traditional SMI, such as low availability, high threshold for participation and substantial delivery costs. Advantages of internet-based SMI (iSMI) are: (1) they are easily accessible at any time and place; (2) anonymity is assured when individuals want to avoid stigmatisation; (3) participants can work at their own pace and review materials as often as they want; (4) such interventions may reach affected employees earlier than traditional mental health services, hence preventing the onset of more severe mental health problems; finally, (5) internet-based interventions are easily scalable, thus 
only a small increase of resources is required for reaching a greater proportion of the eligible population using these interventions. ${ }^{7}$ Internet interventions may also help a population that is not reached by existing approaches.

While there is plenty of evidence for the effectiveness of internet-based interventions in clinical populations, ${ }^{8-10}$ few studies to date have been conducted on interventions that address the management of work-related stress among employees. Results from a few randomised trials provide evidence for the potential of therapist-assisted and coach-assisted SMIs. ${ }^{11-15}$ However, the aforementioned studies evaluated interventions that provided participants with substantial professional support (ranging from approximately 1-14 h of guidance from a mental health expert for each participant). Although this is less resource intensive than most individual stress management interventions, it is still time-consuming. Once developed, the costs of internetbased interventions are substantially linked to professional guidance time. Thus, delivering such interventions with guidance clearly limits the possible reach of such interventions. Moreover, guidance requires professionals who are trained in delivering these interventions. This is not only costly but also makes it more difficult to disseminate these interventions, especially in, for example, low-income and middle-income countries. Thus, from a public health perspective, it would be desirable if no professional guidance was needed in order to achieve clinically relevant effects. Even if pure self-guided interventions should yield lower effects than guided interventions, their potential at a population level might still be higher since more participants can be treated at the same costs. Also, barriers for an effective dissemination are lower.

However, evidence on the effectiveness of pure self-guided intervention for work-related stress is scarce, and the few trials that have been conducted yielded mixed results, ranging from non-significant ${ }^{16}{ }^{17}$ to those producing small effects. ${ }^{18} 19$ Evidence for the long-term effectiveness of self-guided interventions for work-stress is scarce. It remains a challenging task to develop and disseminate internet-based self-guided interventions for work-related stress that require no guidance but produce a clinically relevant outcome.

This study aims at investigating the acceptability and effectiveness of iSMI compared to a 6-month wait-list control group (WLC) on stress, mental health and work-related outcomes in employees with heightened levels of perceived stress.

\section{METHODS}

Design

A two-armed RCT was conducted with two conditions, a selfguided iSMI (GET.ON Stress) and a WLC condition. Both conditions had full access to treatment as usual (TAU). Meta-analyses indicate that short-term effects of face-to-face SMIs range from $\mathrm{d}=0.34^{5}$ to $0.73 .^{6}$ Thus, we included 264 employees. This sample size will allow us to detect an effect size of Cohen's $d=0.35$ based on a power $(1-\beta)$ of 0.80 in a twotailed test with an $\alpha$ of 0.05 .

Participants were primarily recruited via the occupational health programme of a large health insurance company in Germany. Recruitment was directed at the general working population and not restricted to members of the healthcare insurance company. It occurred through announcements on the healthcare insurance company's website, newspaper articles and advertisements in the membership magazine of the insurance company. Moreover, the insurance company's occupational health management workers informed human resource departments of collaborating small-sized and medium-sized companies about the possibility for their employees to participate in the trial.

Assessments took place at baseline (T1), at post-treatment (7 weeks, T2) and at 6 months (T3; see figure 1 for a detailed overview of assessments). The study was approved by the University of Marburg ethics committee. The trial is registered in the German clinical trials register (DRKS00005384).

\section{Inclusion and exclusion criteria}

We included (1) currently employed individuals, (2) above the age of 18 years, (3) with scores $\geq 22$ on the Perceived Stress Scale (PSS-10); ${ }^{20}$ (4) who had internet access and (5) sufficient skills in reading and writing German (self-report). We excluded participants who (1) self-reported to have been diagnosed with psychosis or dissociative symptoms in the past and (2) showed a notable suicidal risk, as indicated by a score higher than 1 on Becks depression inventory item 9 ('I feel I would be better off dead'). The cut-off on the PSS-10 was chosen to select participants with a heightened level of subjective stress as identified by one $\mathrm{SD}(\mathrm{SD}=6.2)$ above the mean (PSS-10=15.3) in a large working population. $^{21}$

\section{Assessment of eligibility and randomisation}

Individuals who applied for study participation received an email with detailed information about the study procedures, and were asked to provide an email address and to complete an online screening questionnaire. Individuals meeting all of the inclusion and none of the exclusion criteria completed the baseline assessment, and were invited to complete the informed consent form. Once the full written informed consent was received, participants entered the study and were randomly allocated to one of the two study conditions. Randomisation took place at a ratio of 1:1 and a block size of 2 . The allocation was performed by an independent third party who did not have any information about the participant. Randomisation was carried out using an automated computer-based random integer generator (Randlist). Other researchers did not have the possibility to bias the randomisation process, because participants were randomised in order of the incoming informed consent form.

Participants were not blinded to study conditions. During the randomisation process, the allocation was concealed from participants, researchers involved in recruitment and e-coaches.

\section{Study conditions}

\section{Intervention condition}

The internet-based intervention GET.ON Stress (for a detailed description see ref. 14) was based on the Lazarus and Folkman $^{22}$ transactional model of stress and its distinction of problem-focused and emotion-focused coping. The intervention was developed using evidence-based material on problem-solving $^{23} \quad 24$ and emotion regulation. ${ }^{25}$ Problem-focused coping is used to actively influence a stress situation in a positive way through the use of cognitive or behavioural efforts. Emotion regulation refers to a variety of processes whereby individuals attempt to control and manage the spontaneous flow of their emotions to accomplish their needs and goals. Emotion-focused coping primarily serves the function of managing difficult emotions such as anger, disappointment, frustration and sadness, in relation to the specific situation. On the one hand, many problems employees often encounter can theoretically be solved. Problem-solving is an evidence-based method for dealing with such problems that has been proven to be successful in improving mental health. ${ }^{24}$ On the other hand, employees are also frequently faced with 


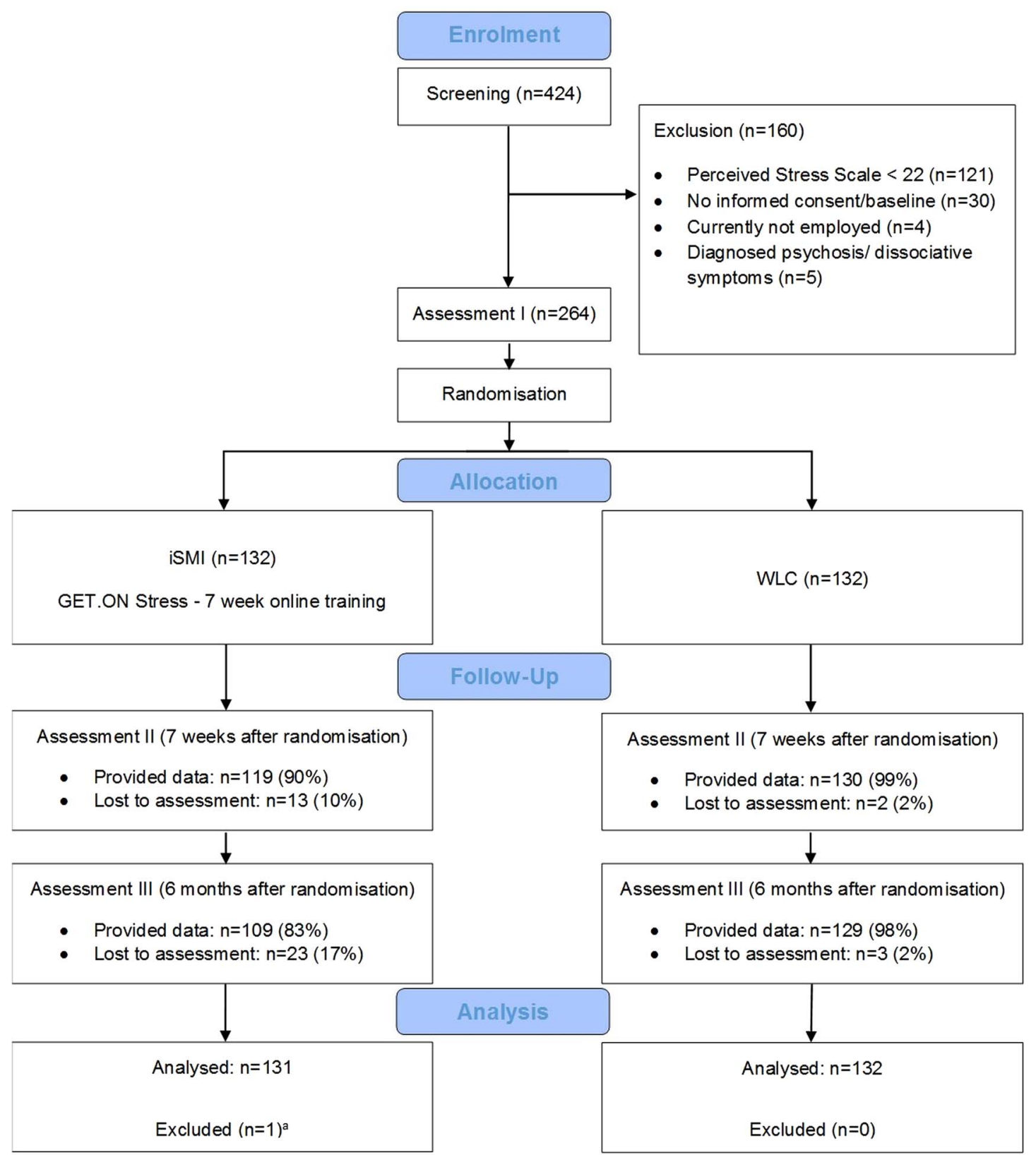

Figure 1 Flow of participants. Note: One participant requested deletion of all data. iSMI, internet-based stress management intervention; WLC, wait-list control group.

situations that require dealing with unsolvable problems; such situations are often associated with strong negative affective reactions and require effective strategies on how to regulate these emotions. A large number of studies indicate that deficits in emotion regulation may be a relevant factor for the development and persistence of mental health symptoms. ${ }^{26} 27$ Emotion regulation skills have been shown to be promising for reducing a broad range of such symptoms. ${ }^{25}$ While problem-focused coping by means of problem-solving techniques is a wellestablished component of most cognitive behavioural therapy (CBT) stress management training, the emotion-focused ways of coping could be regarded as the forgotten component. Only recently has emotion regulation gained more attention and have elaborated concepts been developed. The intervention consists of seven sessions composed of modules for psycho-education (session 1), problem-solving (sessions 2-3), emotion regulation (sessions 4-6), planning for the future (session 7)-and an optional booster session 4 weeks after completion of the iSMI (session 8). Additionally, participants were offered eight optional modules that were integrated into sessions $2-6$, and could be chosen based on individual need and/or preference. These optional modules focused on time management, rumination and worrying, psychological detachment from work, sleep restriction and stimulus control, nutrition and exercise, organisation of breaks during work and social support. Each session could be completed in approximately 45-60 min. We advised the participants to complete at least one and a maximum of two sessions per week. The sessions consisted of texts, exercises and testimonials, and included interactive elements such as audio files and video clips. The participants were encouraged to keep an online stress diary. A strong focus of the intervention was on transferring the newly acquired techniques into daily life (homework 
assignments). The iSMI is adaptive as the content is tailored to the specific needs of the individual participant by continuously asking to choose among various response options. Subsequent content was then tailored to each participant's response (eg, if participants reported that they could not solve the problem they had worked on in the problem-solving module, they were asked to reflect on potential reasons for this; strategies for handling common difficulties were presented, and they were invited to re-adjust their plan, whereas if they indicated to have succeeded, they were invited to train their problem-solving skills on a new problem). If desired, the participants received automatic motivational text messages and small exercises on their mobile phones. These messages aimed at supporting the participants in transferring the training exercises into their daily lives (eg, short relaxation exercises: "Relax the muscles in your hands and arms for $3 \mathrm{~s}$ now. Follow your breathing and each time you breathe out, relax a little more"). The participants had the opportunity to choose between 'light coaching' (1 text message every other day) and 'intensive coaching' (2-3 text messages every day).

\section{Wait-list control group}

Employees in the WLC only received the iSMI 6 months after randomisation but until then, had full access to TAU offered by workplace occupational health management programmes and routine healthcare services.

\section{Primary outcome measure}

\section{Perceived stress}

The primary outcome was level of perceived stress as measured by the 10 -item version of PSS- $10 .^{20}$ The PSS-10 assesses the degree to which people perceive their lives as stressful, particularly regarding how unpredictable, uncontrollable and overloading respondents find their lives (items accessible in online supplementary figure S1). The predictive validity of the PSS was evaluated in a large number of studies, of which the PSS was strongly correlated with the mental component of quality of life and moderately to strongly correlated to depression, as well as moderately correlated with anxiety and emotional exhaustion. ${ }^{28}$ Cronbach's $\alpha$ s for this scale were 0.88 at T2 and 0.92 at T3 in this study. The items were answered on a five-point Likert scale $(0=$ never; $4=$ very often $)$. Accordingly, the sum score ranges from 0 to 40 .

\section{Secondary outcome measures}

In secondary analyses the effect of the below-mentioned outcomes between iSMI and WLC group is reported. The number of items, range of items and reliabilities found at $\mathrm{T} 2$ in this study are shown in parentheses.

\section{Mental health}

Additional mental health outcomes included depression (Center for Epidemiological Studies' Depression Scale, CES-D; ${ }^{29} 20$ items; range $0-60 ; \alpha=0.91$ ), anxiety (anxiety subscale of the Hospital Anxiety and Depression Scales, HADS-A; ${ }^{30} 7$ items; range $0-21 ; \alpha=0.82$ ); insomnia severity (Insomnia Severity Index, ${ }^{31} ; 7$ items; range $0-28 ; \alpha=0.89$ ) and worrying (Penn State Worry Questionnaire Ultra Brief Version-past week, PSWQ-PW; 323 items; range 0-18; $\alpha=0.88$ ).

\section{Work-related health}

Emotional exhaustion (subscale emotional exhaustion of the Maslach Burnout Inventory, MBI- EE; 33 items; range 1-6; $\alpha=0.87$ ), work engagement (Utrecht Work Engagement Scale, $\mathrm{UWES}^{34} 9$ items; range $\left.0-6 ; \alpha=0.94\right)$ and psychological detachment from work (subscale of the Recovery Experience Questionnaire, REQ-PD; ${ }^{35} 4$ items; range $1-5 ; \alpha=0.93$ ) were assessed as work-related outcomes. To assess the number of 'work loss' days (absenteeism from work) and the number of 'work cut-back' days (reduced efficiency at work while feeling ill; presenteeism) we used the items of the Trimbos and Institute of Medical Technology Assessment Cost Questionnaire for Psychiatry (TiC-P-G, ${ }^{36}$ ) in relation to the previous 3 months.

\section{Skills/competencies}

Furthermore, various emotion regulation skills were assessed (subscales comprehension, acceptance and emotional selfsupport of the ERSQ-27 (-C, -A,-SS); ${ }^{37} 9$ items, range 0-4; $\alpha=0.81,0.85,0.88$; subscale emotion regulation skills for general distress of the German Emotion Regulation Skills Questionnaire using the Emotion Specific Version, ERSQ-ES-GD; ${ }^{38} 12$ items, range $0-4 ; \alpha=0.88$ ).

\section{Additional measurements}

Additional questionnaires included demographic variables and client satisfaction (German version of the Client Satisfaction Questionnaire, adapted to the online context, CSQ $-8,{ }^{39} ; 8$ items).

\section{Statistical analyses}

All analyses are reported according to the Consolidated Standards of Reporting Trials (CONSORT) statement, using the intention-to-treat principle (ITT). Additionally, completers-only analyses are reported. Analyses were performed with IBM-SPSS V.22. A significance level of 0.05 (two-sided) was used for all analyses. Multiple imputation was used to handle missing data. Ten single imputations of the missing values were calculated based on the valid data for all outcome measures at all assessment points (T1, T2 and T3) as well as age and gender, and were aggregated into a single overall estimate of the effects of the intervention.

Differences in perceived stress scores between iSMI and WLC groups were assessed using analysis of covariance (ANCOVA) with baseline scores as covariate. Cohen's ds and 95\% CIs were calculated based on the imputed data set comparing the means and SDs of the iSMI and WLC groups at post-test, and at 6-month follow-up. According to Cohen, $\mathrm{d}=0.2$ can be considered a small effect, $\mathrm{d}=0.5$ a medium and $\mathrm{d}=0.8$ a large effect. To determine the numbers of participants achieving a reliable, positive outcome, we coded participants as responders or nonresponders according to the widely used Reliable Change Index (RCI). Hence, participants were considered responders if their PSS-10 score differed more than -5.16 points from baseline to post-assessment and from baseline to 6-month follow-up. Additionally, the numbers needed to treat (NNT) indicating the number of participants that have to be treated to generate one additional treatment response as compared to the control group was calculated. To determine potential negative effects of the intervention we also assessed the number of participants with reliable symptom deterioration according to the RCI. We also calculated a cut-off value for a symptom-free status according to the RCI. Symptom-free status was defined as scoring more than 2 SDs below the mean (T1) of the stressed population (PSS $<16.88)$.

\section{RESULTS \\ Participants}

The enrolment and flow of participants throughout the study is summarised in figure 1. Study drop out was low. Across both 
study groups, 249 (94\%) and 238 (90\%) participants provided follow-up data at 7 weeks, and at 6 months, respectively. In the iSMI group, one participant requested deletion of all data after the study had finished. With regard to missing data, the number of people not providing data significantly differed between iSMI and WLC groups at T2 $\left(\chi^{2}=7.6, p<0.01\right)$ and T3 $\left(\chi^{2}=16.1\right.$, $\mathrm{p}<0.001)$. However, participants who did not provide data at one of the follow-up assessments did not differ to a meaningful extent from participants without missing data on any of the baseline characteristics.

\section{Baseline characteristics}

Table 1 presents the baseline characteristics of the study participants. Employees had an average work experience amount of 18 years $(\mathrm{SD}=10)$. Only $35(13 \%)$ participants out of the entire sample had participated in any traditional mental health/

Table 1 Baseline characteristics

\begin{tabular}{|c|c|c|c|}
\hline Characteristics & $\begin{array}{l}\text { All participants } \\
(n=263)\end{array}$ & $\begin{array}{l}\text { iSMI } \\
(n=131)\end{array}$ & $\begin{array}{l}\text { WLC } \\
(n=132)\end{array}$ \\
\hline \multicolumn{4}{|l|}{ Sociodemographic characteristics } \\
\hline Age (years), M (SD) & $42(9)$ & $41(9)$ & $42(9)$ \\
\hline Gender, female, n (\%) & $188(72)$ & $97(74)$ & $91(69)$ \\
\hline $\begin{array}{l}\text { Married or in a } \\
\text { relationship, } \mathrm{n}(\%)\end{array}$ & $161(61)$ & $81(62)$ & $80(61)$ \\
\hline \multicolumn{4}{|l|}{ Ethnicity, n (\%) } \\
\hline Caucasian/white & $219(83)$ & $107(82)$ & $112(85)$ \\
\hline Asian & $1(1)$ & $1(1)$ & $0(0)$ \\
\hline Prefer not to say & $43(16)$ & $23(18)$ & $20(15)$ \\
\hline \multicolumn{4}{|l|}{ Educational level, n (\%) } \\
\hline Low & $11(4)$ & $6(5)$ & $5(4)$ \\
\hline Middle & $75(29)$ & $39(30)$ & $36(27)$ \\
\hline High & $177(67)$ & $86(66)$ & $91(69)$ \\
\hline \multicolumn{4}{|l|}{ Working characteristics } \\
\hline Full-time, n (\%) & $196(75)$ & $93(71)$ & $103(78)$ \\
\hline Part-time, n (\%) & $62(24)$ & $35(27)$ & $27(21)$ \\
\hline On sick leave, n (\%) & $5(2)$ & $3(2)$ & $2(2)$ \\
\hline $\begin{array}{l}\text { Work experience in years, } \\
\text { M (SD) }\end{array}$ & $18(10)$ & $17(10)$ & $18(9)$ \\
\hline \multicolumn{4}{|l|}{ Working sectors, n (\%) } \\
\hline Economy & $55(21)$ & $22(17)$ & $33(25)$ \\
\hline Service & $50(19)$ & $34(26)$ & $16(12)$ \\
\hline Social & $47(18)$ & $19(15)$ & $28(21)$ \\
\hline Health & $35(13)$ & $17(13)$ & $18(14)$ \\
\hline IT & $22(8)$ & $11(8)$ & $11(8)$ \\
\hline Others & $54(21)$ & $28(21)$ & $26(20)$ \\
\hline \multicolumn{4}{|l|}{ Income in Euros, per year, n (\%) } \\
\hline$<10000$ & $2(1)$ & $1(1)$ & $1(1)$ \\
\hline $10000-30000$ & $68(26)$ & $38(29)$ & $30(23)$ \\
\hline $30000-40000$ & $72(27)$ & $40(31)$ & $32(24)$ \\
\hline $40000-50000$ & $38(14)$ & $12(9)$ & $26(20)$ \\
\hline $50000-60000$ & $23(9)$ & $14(11)$ & $9(7)$ \\
\hline $60000-10000$ & $28(11)$ & $15(12)$ & $13(10)$ \\
\hline$>100000$ & $12(5)$ & $4(3)$ & $8(6)$ \\
\hline Prefer not to say & $20(8)$ & $7(5)$ & $13(10)$ \\
\hline \multicolumn{4}{|l|}{ Experience, n (\%) } \\
\hline Previous health training & $35(13)$ & $14(11)$ & $21(16)$ \\
\hline Previous psychotherapy & $76(29)$ & $37(28)$ & $39(30)$ \\
\hline Current psychotherapy & $25(10)$ & $10(8)$ & $15(11)$ \\
\hline
\end{tabular}

occupational mental health promotion training before. A total of $144(55 \%)$ were first-time-help-seekers for any kind of mental health complaint (ie, never took part in mental health promotion training or psychotherapy). Few participants reported being currently on sick leave $(n=5 ; 2 \%)$. Table 2 shows descriptive data for all outcome variables at all assessment points.

\section{Changes in perceived stress}

As hypothesised, the iSMI group showed lower scores on the primary outcome PSS-10 at post-test $(\mathrm{T} 2 ; \mathrm{F}=62.2, \mathrm{p}<0.001)$ and at the 6-month follow-up (T3; $\mathrm{F}=27.1, \mathrm{p}<0.001)$ as compared with the WLC. A large effect size was observed at post-test ( $\mathrm{d}=0.96 ; 95 \% \mathrm{CI} 0.70$ to 1.21$)$. The effect was moderate to large at the 6-month follow-up $(\mathrm{d}=0.65 ; 95 \%$ CI 0.40 to 0.89 ).

\section{Treatment response}

At post-test, significantly more participants in the iSMI group were classified as responders $\mathrm{n}=77$ (59\%) compared with the WLC $\left(\mathrm{n}=38,29 \% ; \chi^{2}=24.0 ; \mathrm{p}<0.001\right)$. This resulted in an $\mathrm{NNT}=3.3$ (95\% CI 2.4 to 5.4) in order to achieve one additional treatment response as compared to the WLC. Also, at the 6-month follow-up, significantly more participants of the iSMI group achieved response $(\mathrm{n}=81 ; 62 \%)$ compared with the WLC $\left(\mathrm{n}=51 ; 39 \%\right.$; WLC; $\chi^{2}=14.2 ; \mathrm{p}<0.001, \mathrm{NNT}=4.3,95 \%$ CI 2.9 to 8.7 ).

\section{Symptom deterioration}

Fewer participants in the iSMI group experienced a reliable symptom deterioration at T2 $(\mathrm{n}=3 ; 2 \%)$ and T3 $(\mathrm{n}=4 ; 3 \%)$ as compared with the WLC (T2: $n=6,5 \%$; T3: $n=7,5 \%)$, although these differences were not statistically significant.

\section{Close to symptom-free status}

At post-test, a close to symptom-free status was shown by significantly more participants in the iSMI group $(n=50 ; 38 \%)$ as compared with the WLC $\left(\mathrm{n}=17 ; 13 \% ; \chi^{2}=22.2, \mathrm{p}<0.001\right)$, resulting in an $\mathrm{NNT}=4.0$ (95\% CI 2.8 to 6.6). At 6-month follow-up, significantly more participants of the iSMI group $(\mathrm{n}=64 ; 49 \%)$ reached a state of remission as compared with the WLC $\left(\mathrm{n}=35 ; 27 \% ; \chi^{2}=14.0, \mathrm{p}<0.001 ; \mathrm{NNT}=4.5,95 \% \mathrm{CI}\right.$ 3.0 to 9.1$)$.

\section{Secondary outcomes}

Significant effects (all $\mathrm{p}<0.001)$ in favour of the intervention group were found at both assessment points for the mental health-related outcomes of depression, insomnia, anxiety, worrying and quality of life regarding mental health (table 3). Work-related health was found to significantly differ between groups in favour of the intervention group with regard to emotional exhaustion $(\mathrm{p}<0.001)$, psychological detachment from work $(\mathrm{p}<0.001)$, work engagement (only T2; $\mathrm{p}<0.05$ ) and presenteeism $(\mathrm{p}<0.01)$; with regard to emotion regulation, skills and competencies (ie, comprehension, acceptance, selfsupport and regulation of general distress) improved significantly more in the intervention as compared with the control group, at both assessment points (all $\mathrm{p}<0.001)$. The effect sizes ranged from $\mathrm{d}=0.30$ (95\% CI 0.06 to 0.54 ) for presenteeism at $\mathrm{T} 3$ to $\mathrm{d}=0.69(95 \% \mathrm{CI} 0.45$ to 0.94$)$ for worrying at T3 (see table 3 for more details). 
Table 2 Means and SDs for the ITT sample (iSMI: N=131; WLC: N=132) at pre-treatment (T1) and post-treatment (T2), and 6-month follow-up (T3)

\begin{tabular}{|c|c|c|c|c|c|c|c|c|c|c|c|c|}
\hline \multirow[b]{3}{*}{ Outcome } & \multicolumn{4}{|l|}{$\mathrm{T1}$} & \multicolumn{4}{|l|}{$\mathrm{T} 2^{*}$} & \multicolumn{4}{|l|}{ T3* } \\
\hline & \multicolumn{2}{|l|}{ iSMI } & \multicolumn{2}{|l|}{ WLC } & \multicolumn{2}{|l|}{ iSMI } & \multicolumn{2}{|l|}{ WLC } & \multicolumn{2}{|l|}{ iSMI } & \multicolumn{2}{|l|}{ WLC } \\
\hline & M & SD & M & SD & M & SD & M & SD & $M$ & SD & $M$ & SD \\
\hline \multicolumn{13}{|l|}{ Primary outcome } \\
\hline PSS & 25.7 & 5.0 & 26.1 & 4.1 & 18.1 & 5.7 & 23.4 & 5.4 & 17.5 & 6.7 & 21.8 & 6.7 \\
\hline \multicolumn{13}{|l|}{ Mental health } \\
\hline CES-D & 25.1 & 9.31 & 23.9 & 8.3 & 16.1 & 8.7 & 21.4 & 9.1 & 15.2 & 9.0 & 20.2 & 10.0 \\
\hline ISI & 13.0 & 5.6 & 12.8 & 6.0 & 9.3 & 5.2 & 11.2 & 6.5 & 8.0 & 5.1 & 10.3 & 6.0 \\
\hline HADS-A & 11.4 & 3.4 & 11.3 & 3.6 & 8.0 & 3.7 & 9.9 & 3.8 & 7.2 & 3.7 & 9.3 & 4.2 \\
\hline PSWQ & 10.8 & 3.8 & 10.9 & 3.8 & 7.0 & 4.2 & 9.1 & 4.5 & 6.1 & 3.9 & 8.9 & 4.2 \\
\hline SF-12-PH‡ & 49.0 & 9.4 & 46.7 & 9.5 & NA & NA & NA & NA & 51.0 & 6.9 & 48.2 & 9.4 \\
\hline SF-12-MH & 33.2 & 10.0 & 33.5 & 8.3 & NA & NA & NA & NA & 43.2 & 9.9 & 38.3 & 10.1 \\
\hline \multicolumn{13}{|c|}{ Work-related health } \\
\hline MBI-EE & 4.8 & 0.8 & 4.7 & 0.7 & 3.9 & 0.9 & 4.4 & 0.9 & 3.8 & 1.0 & 4.3 & 0.9 \\
\hline UWES & 3.1 & 1.2 & 3.2 & 1.2 & 3.2 & 1.0 & 3.0 & 1.2 & 3.2 & 1.1 & 3.1 & 1.2 \\
\hline REQ-PD $\ddagger$ & 2.3 & 0.9 & 2.3 & 0.9 & 2.9 & 0.9 & 2.5 & 0.9 & 3.0 & 1.0 & 2.5 & 0.9 \\
\hline Absenteeism§ & 5.6 & 12.4 & 6.2 & 12.5 & NA & NA & NA & NA & 3.6 & 9.1 & 4.9 & 12.0 \\
\hline Presenteeism§ & 16.1 & 17.1 & 14.2 & 14.6 & NA & $\mathrm{NA}$ & NA & NA & 7.2 & 9.6 & 10.5 & 12.2 \\
\hline \multicolumn{13}{|c|}{ Skills/competencies } \\
\hline ERSQ-C & 2.4 & 1.0 & 2.5 & 0.9 & 2.8 & 0.7 & 2.5 & 0.8 & 3.0 & 0.7 & 2.6 & 0.9 \\
\hline ERSQ-A $\ddagger$ & 2.0 & 0.9 & 2.1 & 0.8 & 2.6 & 0.7 & 2.2 & 0.9 & 2.8 & 0.8 & 2.3 & 0.9 \\
\hline ERSQ-SS $\ddagger$ & 2.2 & 0.9 & 2.3 & 0.8 & 2.7 & 0.8 & 2.3 & 0.8 & 2.8 & 0.8 & 2.4 & 0.9 \\
\hline ERSQ-GD & 1.8 & 0.6 & 1.8 & 0.6 & 2.3 & 0.6 & 2.0 & 0.6 & 2.5 & 0.6 & 2.1 & 0.6 \\
\hline
\end{tabular}

*Missing data imputed by multiple imputation.

¥Higher scores indicate better outcomes.

§In relation to the previous 3 months.

A acceptance; CES-D, Center for Epidemiological Studies' Depression Scale; C, comprehension; ERSQ, Emotion Regulation Skills Questionnaire; ES-GD, emotion-specific version-general distress; HADS-A, subscale of the Hospital Anxiety and Depression Scales; ISI, Insomnia Severity Index; iSMI, internet-based stress management intervention; ITT, intention-to-treat; MBI-EE, Emotional Exhaustion Subscale of the Maslach Burnout Inventory; NA, not available for this assessment point; PSS-10, Perceived Stress Scale; PSWQ-PW, Penn State Worry Questionnaire-Past Week; REQ-PD, Psychological Detachment Subscale of the Recovery Experience Questionnaire; SF-12 MH, Quality of Life (mental health component); SF-12 PH,

Quality of Life (physical health component); SS, self-support; UWES, Utrecht Work Engagement Scale; WLC, wait-list control group.

\section{Completers-only analysis}

Completers-only analysis revealed similar large effects for the primary outcome at $\mathrm{T} 2 \quad(\mathrm{n}=249 ; \mathrm{F}(1146)=60.6, \mathrm{p}<0.001$; $\mathrm{d}=0.97$; CI 0.71 to 1.23$)$ and T3 $(\mathrm{n}=238 ; \mathrm{F}(1235)=26.4$, $\mathrm{p}<0.001 ; \mathrm{d}=0.67$; CI 0.41 to 0.93 ). Results for secondary outcomes closely mirrored those of the ITT analyses, all significant effects found in the ITT analysis were also effective in the completers-only analysis (data not shown). The only difference was that the effect on work engagement at 6-month follow-up, which was not significant in the ITT analyses, was significant in the completers-only analysis.

\section{Client satisfaction}

Overall, client satisfaction with the training was high. The majority of participants $(95 \%, n=104)$ who indicated their satisfaction $(\mathrm{n}=110)$ were 'satisfied in an overall, general sense' ('very satisfied' or 'mostly satisfied'), rated the training to be of high quality $(95 \%, n=104$; the kind of training they wanted to receive $(93 \%, \mathrm{n}=102)$, met their needs $(93 \%, \mathrm{n}=102$; 'Almost all', 'Most of them') and helped them to deal effectively with problems $(92 \%, n=101 ;)$. Moreover, $83 \%(n=99)$ were satisfied with the amount of help they received), would use the training again if they needed to and $83 \%(n=102)$ would recommend it to a friend in need of similar help.

\section{Non-usage attrition}

On average, participants in the iSMI group completed 4.4 modules $(\mathrm{SD}=2.8)$, which equals $62 \%$ of the intervention, and worked 6.3 weeks $(\mathrm{SD}=6.9$; range $0-34)$ with the intervention. Please see online supplementary table S1 for more detailed information on session completion rates. Linear regression indicated that participants who completed more modules achieved a greater decrease in perceived stress $(B=-0.50, \quad S E=0.17$, $\mathrm{p}<0.01,95 \%$ CI -0.84 to 0.16 ). The regression coefficient suggested that, with each extra module completed, the drop in perceived stress severity from baseline to post-treatment was greater by 0.5 points on the PSS.

\section{Text message coaching}

In order to investigate the incremental value of the SMS coach, we compared in explorative analysis the participants who chose to receive the text message coach $(n=54)$ with those who did not $(n=77)$ on the PSS-10 at T2 (no SMS: $M=18.6, S D=5.9$; with SMS: $M=17.3, S D=5.2$ ) and T3 (no SMS: $M=18.0$, $\mathrm{SD}=7.0$; with SMS: $\mathrm{M}=16.6, \mathrm{SD}=6.2$ ). ANCOVAs indicated that participants who received the text message coach were significantly $(p<0.05)$ less stressed at both follow-ups than those who did not.

\section{DISCUSSION}

Results of this study support the effectiveness of a self-guided SMI for employees not only for the reduction of perceived stress but also for a range of relevant mental health, workrelated health and skill-related outcomes. A 6-month follow-up indicated that the obtained effects were sustained over time. 
Table 3 Results of the ANCOVAs and Cohen's d for the primary and secondary outcome measures (ITT sample) at post-test and 6-month follow-up

\begin{tabular}{|c|c|c|c|c|c|c|}
\hline \multirow[b]{2}{*}{ Outcome } & \multicolumn{3}{|l|}{ T2† between-groups effect } & \multicolumn{3}{|l|}{$\mathrm{T} 3 \mathrm{t}$ between-groups effect } \\
\hline & Difference in means $(95 \% \mathrm{Cl})$ & Cohen's d $(95 \% \mathrm{Cl})$ & $\begin{array}{l}\text { ANCOVA } \\
\text { F(1260) }\end{array}$ & Difference in means $(95 \% \mathrm{Cl})$ & Cohen's d (95\% Cl) & $\begin{array}{l}\text { ANCOVA } \\
\mathrm{F}(1260)\end{array}$ \\
\hline \multicolumn{7}{|l|}{ Primary outcome } \\
\hline PSS & $-5.3(-6.0$ to -4.6$)$ & $0.96(0.70$ to 1.21$)$ & $62.2^{* * *}$ & $-4.3(-5.1$ to -3.5$)$ & $0.65(0.40$ to 0.89$)$ & $27.1^{* * *}$ \\
\hline \multicolumn{7}{|l|}{ Mental health } \\
\hline CES-D & $-5.3(-6.4$ to -4.2$)$ & $0.60(0.35$ to 0.85$)$ & $42.8^{* * *}$ & $-5.0(-6.2$ to -3.9$)$ & $0.52(0.28$ to 0.77$)$ & $27.5^{* * *}$ \\
\hline ISI & $-1.9(-2.6$ to -1.2$)$ & $0.33(0.08$ to 0.57$)$ & $13.4^{* * *}$ & $-2.3(-3.0$ to -1.6$)$ & $0.42(0.18$ to 0.67$)$ & $18.0^{* * *}$ \\
\hline HADS-A & $-1.9(-2.4$ to -1.4$)$ & $0.50(0.25$ to 0.74$)$ & $28.7^{* * *}$ & $-2.1(-2.6$ to -1.6$)$ & $0.53(0.28$ to 0.78$)$ & $24.7^{* * *}$ \\
\hline PSWQ & $-2.1(-2.6$ to -1.6$)$ & $0.48(0.23$ to 0.72$)$ & $22.4^{* * *}$ & $-2.8(-3.3$ to -2.3$)$ & $0.69(0.45$ to 0.94$)$ & $37.4^{* * *}$ \\
\hline SF-12-PH & NA & NA & NA & 2.8 (1.8 to 3.8$)$ & 0.34 (0.10 to 0.58$)$ & 3.9 \\
\hline SF-12-MH & NA & NA & NA & 4.9 (3.7 to 6.1$)$ & $0.49(0.24$ to 0.73$)$ & $19.4^{* * *}$ \\
\hline \multicolumn{7}{|c|}{ Work-related health } \\
\hline MBI-EE & $-0.5(-0.6$ to -0.4$)$ & 0.55 (0.30 to 0.80$)$ & $33.1^{* * *}$ & $-0.5(-0.6$ to -0.4$)$ & 0.53 (0.29 to 0.78$)$ & $25.8^{* * *}$ \\
\hline UWES & $0.2(0.1$ to 0.3$)$ & $0.17(-0.08$ to 0.41$)$ & $5.4^{*}$ & $0.1(-0.1$ to 0.3$)$ & $0.11(-0.13$ to 0.35$)$ & 3.7 \\
\hline REQ-PD & $0.4(0.3$ to 0.5$)$ & $0.44(0.20$ to 0.69$)$ & $15.0^{* * *}$ & $0.5(0.4$ to 0.6$)$ & $0.48(0.23$ to 0.72$)$ & $18.4^{* * *}$ \\
\hline Absenteeism & NA & NA & NA & $-1.3(-2.6$ to 0.0$)$ & $0.12(-0.12$ to 0.36$)$ & 0.9 \\
\hline Presenteeism & NA & NA & NA & $-3.3(-4.6$ to -2.0$)$ & $0.30(0.06$ to 0.54$)$ & $9.3^{* *}$ \\
\hline \multicolumn{7}{|c|}{ Skills/competencies } \\
\hline ERSQ-27-C & $0.3(0.2$ to 0.4$)$ & $0.38(0.14$ to 0.63$)$ & $19.5^{* * *}$ & $0.4(0.3$ to 0.5$)$ & $0.58(0.33$ to 0.83$)$ & $30.0^{* * *}$ \\
\hline ERSQ-27-A & $0.4(0.3$ to 0.5$)$ & $0.49(0.24$ to 0.73$)$ & $21.5^{* * *}$ & $0.5(0.4$ to 0.6$)$ & $0.65(0.40$ to 0.90$)$ & $33.9^{* * *}$ \\
\hline ERSQ-27-SS & $0.4(0.3$ to 0.5$)$ & $0.54(0.29$ to 0.79$)$ & $27.5^{* * *}$ & $0.4(0.3$ to 0.5$)$ & $0.40(0.15$ to 0.64$)$ & $15.6 * * *$ \\
\hline ERSQ-ES-GD & $0.3(0.2$ to 0.4$)$ & $0.62(0.37$ to 0.86$)$ & $33.4^{* * *}$ & $0.4(0.3$ to 0.5$)$ & $0.65(0.40$ to 0.90$)$ & $34.4^{* * *}$ \\
\hline
\end{tabular}

Significance levels used: ${ }^{*} \mathrm{p}<0.05 ;{ }^{* *} \mathrm{p}<0.01 ;{ }^{* * *} \mathrm{p}<0.001$.

$\ddagger$ Controlling for pretreatment scores (T1).

A, acceptance; ANCOVA, analysis of covariance; CES-D, Center for Epidemiological Studies' Depression Scale; C, comprehension; ERSQ, Emotion Regulation Skills Questionnaire; ES-GD, emotion specific version-general distress; HADS-A, subscale of the Hospital Anxiety and Depression Scales; ISI, Insomnia Severity Index; MBI-EE, Emotional Exhaustion Subscale of the Maslach Burnout Inventory; NA, not available for this assessment point; PSS-10, Perceived Stress Scale; PSWQ-PW, Penn State Worry Questionnaire-Past Week; REQ-PD, Psychological Detachment Subscale of the Recovery Experience Questionnaire; SF-12 MH, Quality of Life (mental health component); SF-12 PH, Quality of Life (physical health component); SS, self-support; UWES, Utrecht Work Engagement Scale.

This study supports observations from other studies that internet-based treatments as an occupational mental health intervention is able to reach people who were previously unreached by traditional occupational health approaches. ${ }^{23} 40$

The effects found in the present study compare favourably to results for short-term effects found in the few trials on selfguided iSMIs in employees that have been conducted to date. Previous trials yielded mixed results, ranging from nonsignificant, ${ }^{16} 17$ to those producing small effects. ${ }^{18}$ Our results are comparable to short-term effects found for face-to-face occupational SMIs in the latest meta-analysis on this topic $(d=0.73$ for stress-reduction, $d=0.68$ for anxiety, $d=0.44$ for mental health issues such as depression). ${ }^{6}$ Effects are slightly lower for stress reduction than mean results for CBT-based interventions in this meta-analysis $(\mathrm{d}=1.15$, for stress reduction), but comparable for mental health $(\mathrm{d}=0.71)$, although the CI for the effect sizes found in the present study overlap with those of effects found in that meta-analysis. Comparing longterm effects of the present intervention to meta-analytic findings for face-to-face interventions is not possible as the most recent meta-analysis on this topic ${ }^{6}$ was not able to examine those due to a lack of studies assessing a long-term follow-up.

There may be several potential explanations for finding more favourable effects compared to previous trials on self-guided iSMI. First, the available trials conducted on the topic evaluated internet-based interventions that were developed over a decade ago and therefore were not able to utilise the advantages of recent technological advances, theoretical developments and empirical research. These innovations include, for example, a higher grade of interactivity, the use of prompts and reminders via email or SMS to facilitate behavioural change, and the tailoring of the content of the intervention based on the participants characteristics or input. Second, the intervention was developed based on a clear theoretical framework (Lazarus' transactional stress model) and focused on two core components, problem-solving and emotion regulation techniques. Research on face-to-face stress-management suggests that interventions with fewer treatment components achieve greater effects than those employing more components. ${ }^{6}$ Third, the use of mobile components to foster the transfer of training components into daily life may have reinforced a regular application of the intervention exercises and therefore the efficacy of the training. Explorative analysis on the relationship between the utilisation of the text-message coach and reductions in perceived stress indeed suggests a potential incremental value of the textmessage coach. However, randomised trials comparing the intervention with the text-message coach to the same intervention without the mobile components are needed to confirm such an assumption. Fourth, we excluded over $n=120$ participants due to their low levels of stress and included only employees with highly elevated symptoms of stress, which may have facilitated finding stronger effects compared to other studies with different inclusion criteria. However, according to Hobfoll ${ }^{41}$ highly stressed people compared to those with low stress face greater loss of resources, are more vulnerable to further deterioration and have to invest more energy to lower their level of stress. The mean baseline level of depression (ie, $>3$ points above the cut-off (CES-D >22), ${ }^{42}$ for clinical relevant symptoms of depression) and anxiety ( $>3$ points above the cut-off (HADS-A $>8$ ) for clinical relevant symptoms of anxiety), ${ }^{43}$ also indicate a 
highly distressed sample. Thus, the effects found here with highly impaired people are especially noteworthy and encouraging. However, we do not have any data to support these assumptions and future empirical studies are needed to disentangle strategies that are most promising in improving the effectiveness of iSMIs.

This study has the following limitations. First, the elaborated study inclusion process typical to an RCT (ie, completion of two self-report assessments, sending of informed consent) might have led to greater inclusion of above-average motivated employees than one could expect outside of the controlled research context. This is a common limitation in RCTs on psychological interventions but may have a particularly high impact on the results of trials on self-guided interventions. As a result, the findings might not generalise to iSMIs without such an inclusion process. Second, potential negative effects, especially for participants not benefiting from the intervention, such as reduced motivation to engage in psychological interventions in the future, should be assessed in future studies. ${ }^{44}$ Although the present trial clearly indicated that self-guided iSMIs can result in substantial benefits for stressed employees, it may very well be the case that employees are less willing to participate in an intervention if no support from a healthcare professional is provided compared to interventions with professional support. If this is the case, lower overall effects in the target population would result. Thus, future studies should compare the acceptability, effectiveness and cost-effectiveness of different guidance formats for iSMI. ${ }^{45}$ Third, because an open recruitment strategy was used, we cannot draw any conclusions about the reach of the intervention. Future studies should assess in detail what percentage and what kind of employees are indeed willing to utilise such an intervention. Finally, we only included individuals with substantial levels of self-reported stress as identified by one SD above the mean found in a large working population-thus, our results are only valid for this specific target population and do not generalise to less impaired employees. Based on these inclusion criteria we excluded a substantial number of employees $(\mathrm{N}=122)$ who were motivated to participate in the intervention. Given that in routine occupational programmes participants are often not selected based on a pre-defined minimum stress level on a standardised scale, future studies should evaluate the intervention without applying such a cut-off.

In conclusion, iSMI interventions could be an acceptable and effective approach to reduce the negative consequences associated with work-related stress. ${ }^{47}$ Future studies should evaluate the cost-effectiveness of internet-based occupational health interventions including studies that compare guided iSMI to self-help and face-to-face approaches.

Contributors $\mathrm{DDE}, \mathrm{EH}, \mathrm{DL}, \mathrm{HR}, \mathrm{PC}, \mathrm{MB}, \mathrm{BF}$ and $\mathrm{PC}$ contributed to the design of the study. EL, DDE, DL, HR and MB developed the intervention content. DDE and EL performed the outcome analyses. DDE drafted the first proof of the manuscript and integrated co-author comments and edits. All the authors contributed to the further writing of the manuscript and approved the final manuscript.

Funding The BARMER GEK and European Union funded this study (EU EFRE: ZW6-80119999, CCI 2007DE161PR001).

Competing interests DDE and HR are members of the board of the International Society for Research on Internet Interventions. DDE, MB, BF and DL are stakeholders in the 'Institute for Online Health Trainings', which aims to transfer scientific knowledge related to the present research into routine health care.

Patient consent Obtained.

Ethics approval University of Marburg ethics committee.

Provenance and peer review Not commissioned; externally peer reviewed.

Data sharing statement The data set is available from DDE.

\section{REFERENCES}

1 Åkerstedt T, Orsini N, Petersen $\mathrm{H}$, et al. Predicting sleep quality from stress and prior sleep — a study of day-to-day covariation across six weeks. Sleep Med 2012;13:674-9.

2 Richardson S, Shaffer JA, Falzon L, et al. Meta-analysis of perceived stress and its association with incident coronary heart disease. Am J Cardiol 2012;110:1711-16.

3 Stansfeld S, Candy B. Psychosocial work environment and mental health-a meta-analytic review. Scand J Work Environ Health 2006;32:443-62. http://www. ncbi.nlm.nih.gov/pubmed/17173201 (accessed 13 Jan 2015).

4 Kalia M. Assessing the economic impact of stress - the modern day hidden epidemic. Metab Clin Exp 2002;51:49-53. (accessed 3 Mar 2014).

5 Van der Klink JJ, Blonk RW, Schene AH, et al. The benefits of interventions for work-related stress. Am J Public Health 2001;91:270-6. (accessed 3 Mar 2014).

6 Richardson KM, Rothstein HR. Effects of occupational stress management intervention programs: a meta-analysis. J Occup Health Psychol 2008;13:69-93.

7 Ebert DD, Lehr D, Boß L, et al. Efficacy of an internet-based problem-solving training for teachers: results of a randomized controlled trial. Scand I Work Environ Health 2014:40:582-96.

8 Hedman E, Ljótsson B, Lindefors N. Cognitive behavior therapy via the Internet: a systematic review of applications, clinical efficacy and cost-effectiveness. Expert Rev Pharmacoecon Outcomes Res 2012;12:745-64.

9 Riper $\mathrm{H}$, Blankers $\mathrm{M}$, Hadiwijaya $\mathrm{H}$, et al. Effectiveness of guided and unguided low-intensity internet interventions for adult alcohol misuse: a meta-analysis. PLOS ONE 2014:9:e99912.

10 Ebert DD, Zarski AC, Christensen $\mathrm{H}$, et al. Internet and computer-based cognitive behavioral therapy for anxiety and depression in youth: a meta-analysis of randomized controlled outcome trials. PLOS ONE 2015;10:e0119895.

11 Ly KH, Asplund K, Andersson G. Stress management for middle managers via an acceptance and commitment-based smartphone application: a randomized controlled trial. Internet Interv 2014;1:95-101.

12 Ruwaard J, Lange A, Bouwman $\mathrm{M}$, et al. E-mailed standardized cognitive behavioural treatment of work-related stress: a randomized controlled trial. Cogn Behav Ther 2007:36:179-92.

13 Aikens KA, Astin J, Pelletier KR, et al. Mindfulness goes to work: impact of an online workplace intervention. J Occup Environ Med 2014;56:721-31.

14 Heber E, Ebert DD, Lehr D, et al. Efficacy and cost-effectiveness of a web-based and mobile stress-management intervention for employees: design of a randomized controlled trial. BMC Public Health 2013;13:655.

15 Heber $E$, Lehr $D$, Ebert $D$, et al. Web-based and mobile stress management intervention for employees: results of a randomised controlled trial. J Med Internet Res 2016;18:e21

16 Shimazu A, Kawakami N, Irimajiri $\mathrm{H}$, et al. Effects of web-based psychoeducation on self-efficacy, problem solving behavior, stress responses and job satisfaction among workers: a controlled clinical trial. J Occup Health 2005;47:405-13. (accessed 3 Mar 2014)

17 Yamagishi M, Kobayashi T, Nakamura Y. Effects of web-based career identity training for stress management among Japanese nurses: a randomized control trial. J Occup Health 2008;50:191-3. (accessed 19 Dec 2014).

18 Billings DW, Cook RF, Hendrickson A, et al. A web-based approach to managing stress and mood disorders in the workforce. J Occup Environ Med 2008;50:960-8.

19 Hasson D, Anderberg UM, Theorell T, et al. Psychophysiological effects of a web-based stress management system: a prospective, randomized controlled intervention study of IT and media workers [ISRCTN54254861]. BMC Public Health 2005;5:78.

20 Cohen S, Kamarck T, Mermelstein R. A global measure of perceived stress. J Health Soc Behav 1983;24:385-96. (accessed 9 Mar 2014).

21 Lesage FX, Berjot S, Deschamps F. Psychometric properties of the French versions of the Perceived Stress Scale. Int J Occup Med Environ Health 2012;25:178-84.

22 Lazarus RS, Folkman S. Stress, appraisal, and coping. New York, NY: Springer, 1984. http://www.springerpub.com/stress-appraisal-and-coping.html (accessed 22 Feb 2015)

23 Ebert DD, Lehr D, Boß L, et al. Efficacy of an internet-based problem-solving training for teachers: results of a randomized controlled trial. Scand J Work Env Heal 2014:40:582-96.

24 Malouff JM, Thorsteinsson EB, Schutte NS. The efficacy of problem solving therapy in reducing mental and physical health problems: a meta-analysis. Clin Psychol Rev 2007;27:46-57

25 Berking $M$, Ebert DD, Cuijpers $P$, et al. Emotion regulation skills training enhances the efficacy of inpatient cognitive behavioral therapy for major depressive disorder: a randomized controlled trial. Psychother Psychosom 2013;82:234-45.

26 Wirtz CM, Radkovsky A, Ebert DD, et al. Successful application of adaptive emotion regulation skills predicts the subsequent reduction of depressive symptom severity but neither the reduction of anxiety nor the reduction of general distress during the treatment of major depressive disorder. PLOS ONE 2014;9:e108288.

27 Aldao A, Nolen-Hoeksema S, Schweizer S. Emotion-regulation strategies across psychopathology: a meta-analytic review. Clin Psychol Rev 2010;30:217-37.

28 Lee EH. Review of the psychometric evidence of the perceived stress scale. Asian Nurs Res (Korean Soc Nurs Sci) 2012;6:121-7. 
29 Hautzinger M, Bailer M. Allgemeine Depressions Skala. Manual. Göttingen: Beltz Test GmbH, 1993. http://www.psycontent.com/content/3×081j2m2732r345/ (accessed 16 Mar 2014)

30 Hermann-Lingen C, Buss U, Snaith R. Hospital Anxiety and Depression ScaleDeutsche Version (HADS-D). 3rd edn. Bern: Huber Hans, 2011.

31 Morin CM, Belleville G, Bélanger L, et al. The insomnia severity index: psychometric indicators to detect insomnia cases and evaluate treatment response. Sleep 2011;34:601-8. http://www.ncbi.nlm.nih.gov/pmc/articles/PMC3079939/ (accessed 16 Mar 2014).

32 Stöber J. PSWQ-PW: Penn State Worry Questionnaire-Past Week. In: Brähler E, Schumacher J, Strauß B, eds. Diagnostische Verfahren in der Psychotherapie. Hogrefe, Göttingen, Germany: 2002:291-4.

33 Maslach C, Jackson S E, Leiter M P. The Maslach Burnout Inventory (3rd ed). Palo Alto, CA: Consulting Psychologists Press. Palo Alto, CA: Consulting Psychologists Press, 1996.

34 Schaufeli W, Bakker A. Utrecht work engagement scale: test manual. Utrecht, The Netherlands: Departmant of Psychology, Utrecht University, 2003.

35 Sonnentag S, Fritz C. The Recovery Experience Questionnaire: development and validation of a measure for assessing recuperation and unwinding from work. J Occup Health Psychol 2007;12:204-21.

36 Bouwmans $C$, De Jong $K$, Timman $R$, et al. Feasibility, reliability and validity of a questionnaire on healthcare consumption and productivity loss in patients with a psychiatric disorder (TiC-P). BMC Health Serv Res 2013;13:217.

37 Berking M, Znoj H. Entwicklung und Validierung eines Fragebogens zur standardisierten Selbsteinschätzung emotionaler Kompetenzen (SEK-27). Zeitschrift für Psychiatr Psychol und... Published Online First: 2008. http://www.psycontent. com/index/K300NR32641827R4.pdf (accessed 16 Mar 2014).

38 Ebert DD, Christ O, Berking M. Entwicklung und Validierung eines Fragebogens zur emotionsspezifischen Selbsteinschätzung emotionaler Kompetenzen (SEK-ES) Diagnostica Published Online First: 2013. http://www.psycontent.com/index/ F224M028N22W4175.pdf (accessed 16 Mar 2014).
39 Attkisson CC, Zwick R. The client satisfaction questionnaire. Psychometric properties and correlations with service utilization and psychotherapy outcome. Eval Program Plann 1982;5:233-7. http://www.ncbi.nlm.nih.gov/pubmed/10259963 (accessed 20 Feb 2014).

40 Thiart $H$, Lehr $D$, Ebert DD, et al. Log in and breathe out: internet-based recovery training for sleepless employees with work-related strain-results of a randomized controlled trial. Scand J Work Environ Health 2015;41:164-74.

41 Hobfoll SE. The influence of culture, community, and the nested-self in the stress process: Advancing Conservation of Resources theory. 2001, volume 50, issue 3, pages 337-421, doi:10.1111/1464-0597.00062

42 Hautzinger $M$, Bailer $M$, Hofmeister $D$, et al. Allgemeine Depressionsskala (ADS). Manual [Center for Epidemiological Studies Depression Scale (CES-D; Radloff, L.S., 1977)_German version with new Norms. 2nd edn. Hogrefe, 2012. http://www. zpid.de/psychologie/PSYNDEX.php? search=psychauthors\&id=9006484 (accessed 9 Dec 2014).

43 Bjelland I, Dahl AA, Haug TT, et al. The validity of the Hospital Anxiety and Depression Scale. An updated literature review. J Psychosom Res 2002; 52:69-77. http://www.ncbi.nlm.nih.gov/pubmed/11832252 (accessed 6 Nov 2013).

44 Rozental A, Andersson G, Boettcher J, et al. Consensus statement on defining and measuring negative effects of internet interventions. Internet Interv 2014;1:12-19.

45 Ebert DD, Berking M, Cuijpers $P$, et al. Increasing the acceptance of internet-based mental health interventions in primary care patients with depressive symptoms. A randomized controlled trial. J Affect Disord 2015;176:9-17.

46 Boß L, Lehr D, Berking M, et al. Evaluating the (cost-)effectiveness of guided and unguided Internet-based self-help for problematic alcohol use in employees-a three arm randomized controlled trial. BMC Public Health 2015;15:1043.

47 Ebert DD, Berking M, Thiart $\mathrm{H}$, et al. Restoring depleted resources: Efficacy and mechanisms of change of an internet-based unguided recovery training for better sleep and psychological detachment from work. Health Psychol 2015;34 (Suppl):1240-51. 